support from: Amgen, BMS, Janssen, Roche, UCB, AbbVie, Pfizer, Merck, Celgene, Sanofi, Lilly, Novartis., Carol Hitchon Grant/research support from: Pfizer and UCB Canada, Glen Hazlewood: None declared, Edward Keystone Speakers bureau: Amgen, AbbVie, Bristol-Myers Squibb, F. Hoffmann-La Roche Inc., Janssen Inc., Merck, Pfizer Pharmaceuticals, Sanofi Genzyme, UCB, Consultant of:: AbbVie, Amgen, AstraZeneca Pharma, Bristol-Myers Squibb Company, Celltrion, Myriad Autoimmune, F. Hoffmann-La Roche Inc, Genentech Inc, Gilead, Janssen Inc, Lilly Pharmaceuticals, Merck, Pfizer Pharmaceuticals, Sandoz, Sanofi-Genzyme, Samsung Bioepsis, Grant/research support from: AbbVie, Amgen, Gilead Sciences, Lilly Pharmaceuticals, Merck, Pfizer Pharmaceuticals, PuraPharm, Sanofi, Orit Schieir: None declared, Carter Thorne Speakers bureau: Medexus/ Medac, Consultant of: Abbvie, Centocor, Janssen, Lilly, Medexus/Medac, Pfizer, Grant/research support from: Amgen, Pfizer, Abbvie, Celgene, CaREBiodam, Novartis, Diane Tin: None declared, Marie-France Valois: None declared, Vivian Bykerk Consultant of: Amgen, BMS, Gilead, Sanofi-Genzyme/Regeneron, Scipher, Pfizer Pharmaceuticals, UCB, NIH, Lillian Barra: None declared DOI: 10.1136/annrheumdis-2021-eular.1835

\section{POS0532 AVERAGE PREDNISOLONE DOSE OF ONLY 1 MG PER DAY WAS RISK FACTOR FOR CLINICAL FRACTURES IN PATIENTS WITH RHEUMATOID ARTHRITIS - NINE- YEAR FINDINGS OF THE TOMORROW STUDY}

H. Yoshimura ${ }^{1}$, T. Koike ${ }^{2,3}$, K. Mamoto $^{4}$, Y. Sugioka ${ }^{2}$, T. Okano ${ }^{4}$, M. Tada $^{5}$, K. Inui ${ }^{6}$, H. Nakamura ${ }^{4} .{ }^{1}$ Izumi City General Hospital, Orthopaedic Surgery, Osaka, Japan; ${ }^{2}$ Center for Senile Degenerative Disorders (CSDD), Osaka City University Medical School, Orthopaedic surgery, Osaka, Japan; ${ }^{3}$ Search Institute for Bone and Arthritis Disease (SINBAD), Orthopaedic Surgery, Wakayama, Japan; ${ }^{4}$ Osaka City University Medical School, Orthopaedic Surgery, Osaka, Japan; ${ }^{5}$ Osaka City General Hospital, Orthopaedic Surgery, Osaka, Japan; ${ }^{6}$ Osaka Saiseikai Nakatsu Hospital, Orthopaedic Surgery, Osaka, Japan

Background: Previous cohort studies showed that the use of prednisolone (PSL) was a risk factor for clinical fractures in patients with rheumatoid arthritis (RA). However, there are few reports on relationship between PSL dose and clinical fractures. Objectives: The present study aimed to determine the effect of PSL dose on the incidence of clinical fractures in the RA patients treated with PSL.

Methods: We evaluated anthropoetric parameters, bone mineral density (BMD), disease activity score 28-erythrocyte sedimentation rate (DAS28-ESR), RA medication (methotrexate (MTX) dose, use of biologic disease modified anti-rheumatic-drugs (bDMARDs), and PSL dose) and the incidence of clinical fractures during nine years in RA patients who participant the TOMORROW study (UMIN000003876), which is a 10-years prospective cohort study. Data on clinical fracture was self-reported on the questionnaires. In this analysis, the data of RA patients treated with PSL at least once during nine-year period were evaluated. We analyzed the average dose of PSL until the incidence of the clinical fractures. The risk factor for clinical fractures were analyzed by using Cox proportional hazard model with adjustment for age, sex, body mass index (BMI), and smoking history. Results: We analyzed the data of 67 RA patients treated with PSL. Among them, median age was 61.8 year, 56 patients $(83.6 \%)$ were female, 47 patients $(70.1 \%)$ were never smoker and median disease duration was 12.1 year. The number of patients treated with PSL at baseline was 48 (69.1\%). During 9 years, 23 clinical fractures were observed in 67 patients, and the incidence of clinical fracture was $0.046 /$ person-year. In 19 patients, who were not treated with PSL at baseline but treated with PSL at least once during 9 years, 5 clinical fractures were observed. In 67 RA patients, Cox proportional hazard analysis revealed that baseline disease activities, BMD at thoracic vertebrae and medication were not significant risk factors for clinical fractures. However, average PSL dose of more than only $1 \mathrm{mg} /$ day was a significant risk factor for the incidence of clinical fracture (hazard ratio $(\mathrm{HR}): 2.80 ; \mathrm{p}=0.03$ ) (Table 1).

Table 1. Adjusted hazard ratio for clinical fractures in patients with rheumatoid arthritis treated with PSL.

\begin{tabular}{|c|c|c|c|}
\hline & $\begin{array}{l}{ }^{*} \text { Adjusted Hazard } \\
\text { ratio }\end{array}$ & $\begin{array}{l}95 \% \text { Confidence } \\
\text { interval }\end{array}$ & $\begin{array}{l}P \\
\text { value }\end{array}$ \\
\hline CRP (mg/dL) & 1.29 & $0.88-1.91$ & 0.19 \\
\hline $\mathrm{RF}(\mathrm{IU} / \mathrm{mL})$ & 0.99 & $0.99-1.00$ & 0.07 \\
\hline ACPA (U/mL) & 0.99 & $0.98-1.00$ & 0.18 \\
\hline DAS28-ESR & 0.99 & $0.71-1.39$ & 0.97 \\
\hline BMD at thoracic vertebrae $\left(\mathrm{mg} / \mathrm{cm}^{2}\right)$ & 0.02 & $0.00-1.00$ & 0.05 \\
\hline bDMARDs use & 0.55 & $0.23-1.32$ & 0.18 \\
\hline Bisphosphonate use & 2.33 & $0.95-5.71$ & 0.07 \\
\hline average dose of MTX (mg/week) & 1.02 & $0.92-1.12$ & 0.74 \\
\hline average score of DAS28-ESR & 1.15 & $0.76-1.75$ & 0.52 \\
\hline average dose of PSL more than $1 \mathrm{mg} /$ day & 2.8 & $1.09-7.24$ & 0.03 \\
\hline
\end{tabular}

*Hazard ratio was adjusted for age, sex, body mass index (BMI), and smoking history. RF, Rheumatoid factor; ACPA, Anti-cyclic citrullinated peptide antibody; DAS28-ESR, disease activity score 28-erythrocyte sedimentation rate; BMD, Bone mineral density; bDMARDs, biologic disease modified anti-rheumatic-drugs; MTX, methotrexate; PSL, prednisolone.
Conclusion: In RA patients treated with PSL, average PSL dose of only $1 \mathrm{mg}$ day significantly increased the risk for the incidence of clinical fractures. Even for established RA patients, continuous use or initiation of low PSL dose was apparently significant risk factor for clinical fractures.

Disclosure of Interests: Hitoshi Yoshimura: None declared, Tatsuya Koike Grant/ research support from: Takeda Pharmaceutical, Mitsubishi Tanabe Pharma Corporation, Chugai Pharmaceutical, Eisai, Abbott Japan, Teijin Pharma, Banyu Pharmaceutical and Ono Pharmaceutical, Kenji Mamoto: None declared, Yuko Sugioka: None declared, Tadashi Okano: None declared, Masahiro Tada: None declared, Kentaro Inui Grant/research support from: Janssen Pharmaceutical K.K. and Astellas Pharma Inc, Hiroaki Nakamura: None declared DOI: 10.1136/annrheumdis-2021-eular.1868

\section{\begin{tabular}{|l|l}
\hline POS0533 & EFFECT OF CAROTID ULTRASOUND ON THE
\end{tabular} ACHIEVEMENT OF LDL-CHOLESTEROL TARGETS IN THE ROUTINE CLINICAL CARE OF PATIENTS WITH RHEUMATOID ARTHRITIS}

I. Ferraz-Amaro $^{1}$, A. Corrales ${ }^{2}$, N. Vegas-Revenga ${ }^{2}$, B. Atienza-Mateo ${ }^{2}$, V. Portilla ${ }^{2}$, R. Blanco ${ }^{2}$, J. Llorca ${ }^{2}$, M. A. González-Gay ${ }^{2} .{ }^{1}$ Hospital Universitario de Canarias, Division of Rheumatology, Santa Cruz de Tenerife, Spain; ${ }^{2}$ Hospital Universitario Marqués de Valdecilla, Division of Rheumatology, Santander, Spain

Background: Cardiovascular disease (CVD) risk in patients with rheumatoid arthritis (RA) is substantially elevated compared to the general population. In RA as in the general population, CVD control includes the detection and treatment of $\mathrm{CV}$ risk factors based, among others, on blood pressure, smoking, diabetes, and lipid profiles. Little is known about the real impact of the use of carotid ultrasound in the prevention of CVD in patients with RA.

Objectives: To determine whether the use of carotid ultrasound in the routine clinical care of patients with RA can improve the achievement of LDL-cholesterol targets over time.

Methods: We conducted a retrospective, real-world study of 327 RA patients in which a carotid ultrasound was performed as part of routine clinical care. Participants were followed from 2012 to 2018. LDL-c levels were measured before and after the carotid ultrasound intervention. The achievement of the LDL-c goals recommended by the international guidelines was compared before and after the carotid ultrasound. Predictive factors of achievement of LDL-cholesterol targets were studied.

Results: When considering the 2010 EULAR RA SCORE risk categories, serum LDL-c levels in the moderate CV risk category was significantly lower when follow-up finished $(126 \pm 33$ to $109 \pm 29 \mathrm{mg} / \mathrm{dl}, \mathrm{p}=0.000)$ (Table 1). This was not the case for other CV risk categories. Similarly, LDL-C goal attainment in the moderate CV risk category was significant higher at the end of the study compared to baseline. Based on the 2016 European Society of Cardiology LDL-c targets, the achievement of a LDL-cholesterol inferior to $115 \mathrm{mg} / \mathrm{dl}$ for the moderate $\mathrm{CV}$ risk category significantly increased from 35 to $64 \%(p=0.000)$ after follow-up. However, significant changes were not observed in this regard for the low, high, and very-high $\mathrm{CV}$ risk categories.

Table 1. LDL cholesterol serum levels and LDL goals differences between baseline and final follow-up

\begin{tabular}{lccccccccc}
\hline & \multicolumn{4}{c}{ LDL, mg/dl } & \multicolumn{3}{c}{ LDL (mg/dl) ESC 2016 goals } \\
\cline { 2 - 10 } & $n$ & $\%$ & Baseline & $\begin{array}{c}\text { Final } \\
\text { follow-up }\end{array}$ & Goal & Baseline & $\begin{array}{c}\text { Final } \\
\text { follow-up }\end{array}$ & $p$ \\
\hline 2010 EULAR RA SCORE & & & & & & & & \\
Low & 120 & 37 & $116 \pm 37$ & $119 \pm 32$ & 0.081 & $<130$ & $68 \%$ & $66 \%$ & 0.99 \\
Moderate & 195 & 60 & $126 \pm 33$ & $109 \pm 29$ & $\mathbf{0 . 0 0 0}$ & $<115$ & $35 \%$ & $64 \%$ & $\mathbf{0 . 0 0 0}$ \\
High & 7 & 2 & $121 \pm 26$ & $104 \pm 22$ & 0.14 & $<100$ & $14 \%$ & $33 \%$ & 0.99 \\
Very High & 5 & 0 & $131 \pm 41$ & $115 \pm 46$ & 0.080 & $<70$ & $0 \%$ & $20 \%$ & 0.99
\end{tabular}

ESC: European Society of Cardiology; EULAR: European League Against Rheumatism SCORE: Systematic COronary Risk Evaluation; RA: Rheumatoid Arthritis; LDL: Iow-density lipoprotein. Significant ' $p$ ' value are depicted in bold.

When patients with RA, regardless the SCORE risk they had, were divided between those that attained LDL-cholesterol target or not, those that achieved their LDL goal, were more frequently female and had less diabetes mellitus. Moreover, the probability of achieving LDL-cholesterol goal was higher in those with an inferior total cholesterol, LDL-c and atherogenic index at baseline. Interestingly, no differences were observed in both populations regarding the baseline use of statins, aspirin or hypertension treatment at baseline. Concerning disease related data when study started, no differences about disease duration and activity or treatments were observed between dose that reached LDL-cholesterol goal and those that did not.

Those that achieved LDL-c objectives had a higher decrease in smoking quit and a superior control of hypertension. Besides, in those that attained objectives a 
superior increase in statins prescription was observed ( $36 \%$ vs. $13 \%, p=0.000)$, as well in hypertension and aspirin treatment. However, no differences were noticed in the changes in disease-related data that occur during the time the study was carried out.

Conclusion: This real-world study demonstrates that incorporating carotid ultrasound into routine clinical practice in RA patients is effective in achieving LDL-cholesterol targets for the prevention of cardiovascular disease. This effect is not only mediated by the increase in the prescription of statins, but also by the better control of other cardiovascular risk factors that occurred after carotid ultrasound evaluation.

Disclosure of Interests: None declared

DOI: 10.1136/annrheumdis-2021-eular.1883

\section{POS0534 $\quad$ THE PREVALENCE OF HYPERTENSION AND ITS POTENTIAL ASSOCIATED FACTORS IN CHINESE PATIENTS WITH RHEUMATOID ARTHRITIS}

Y. W. Zou ${ }^{1}$, C. Chen ${ }^{2}$, J. Lin ${ }^{1}$, J. D. Ma ${ }^{2}$, Y. Y. Zou ${ }^{1}$, L. Dai ${ }^{1}{ }^{1}$ Sun Yat-Sen Memorial Hospital, Sun Yat-Sen University, Department of Rheumatology, Guangzhou, China; ${ }^{2}$ Sun Yat-Sen Memorial Hospital, Sun Yat-Sen University, Department of Rheumatology, Guangzhou, China

Background: Cardiovascular disease (CVD) is the leading cause of mortality in patients with rheumatoid arthritis (RA), and hypertension is a modifiable risk-factor for CVD. In addition, comorbidities may shorten the life span of RA patients, which appears to be the consequence of an increased prevalence of CVD. RA patients with comorbidities might not be given the equal treatment in daily practice compared with patients without comorbidities.

Objectives: To investigate the prevalence of hypertension and its associated factors in Chinese RA patients.

Methods: Consecutive patients with RA were recruited from August 2015 to September 2019 at Department of Rheumatology, Sun Yat-sen Memorial Hospital. Demographic and clinical characteristics were collected including indicators of disease activity, functional assessment and radiographic assessment and CV-related comorbidities.

Results: There were 674 RA patients recruited with $82.3 \%$ female and mean age $49.9 \pm 13.0$ years. The prevalence rate of hypertension in RA patients was $32.9 \%$, followed by dyslipidemia $(9.9 \%)$, type 2 diabetes $(8.8 \%)$, hyperuricemia $(8.5 \%)$, fatty liver disease $(8.0 \%)$, CVD $(6.2 \%)$ and chronic kidney disease $(3.3 \%)$. The prevalence of hypertension was $38.7 \%$ and $31.7 \%$ in male and female RA patients respectively $(P=0.144)$. Further age stratification showed no hypertension in RA patients at $16-19$ years, $2.0 \%$ at $20-29$ years and $8.1 \%$ at $30-39$ years. The prevalence of hypertension greatly increased after 40 years old with $24.5 \%, 38.6 \%, 54.9 \%$, and $63.6 \%$ in RA patients of $40-49,50-59,60-69, \geq 70$ years old respectively, and there was no significant difference between male and female RA patients of different age (Figure 1). Compared with those without hypertension, RA patients with hypertension had advanced age (57.3 \pm 9.3 years vs. $46.3 \pm 13.0$ years), long-standing disease duration (median 60 months vs. 48 months), higher disease activity indicators [including PtGA (median 4 vs. 3), PrGA (median 3 vs. 3) and ESR (median $33 \mathrm{~mm} / \mathrm{h}$ vs. $27 \mathrm{~mm} / \mathrm{h}$ )], higher functional indicator [HAQ-DI (median 0.25 vs. 0.13 )], worse joint destruction [JE subscore (median 10 vs. $6)$ ] and higher proportions of comorbidities [including type 2 diabetes (14.9\% vs. $5.8 \%)$, hyperuricemia $(13.1 \%$ vs. $6.1 \%)$, chronic kidney disease $(6.3 \%$ vs. $1.8 \%$ ), dyslipidemia (15.8\% vs. $7.1 \%)$, CVD (10.4\% vs. $4.2 \%$ ) and fatty liver disease $(13.5 \%$ vs. $5.3 \%)$, all $P<0.005$ ]. Multivariate logistic regression analysis showed that comorbidities including hyperuricemia $(O R=1.977,95 \% \mathrm{Cl}$ : 1.002-3.900), dyslipidemia $(O R=1.903,95 \% \mathrm{Cl}: 1.102-3.288)$ and fatty liver disease $(O R=2.335,95 \% \mathrm{Cl}: 1.278-4.265)$ were associated factors of hypertension after adjustment for age and gender
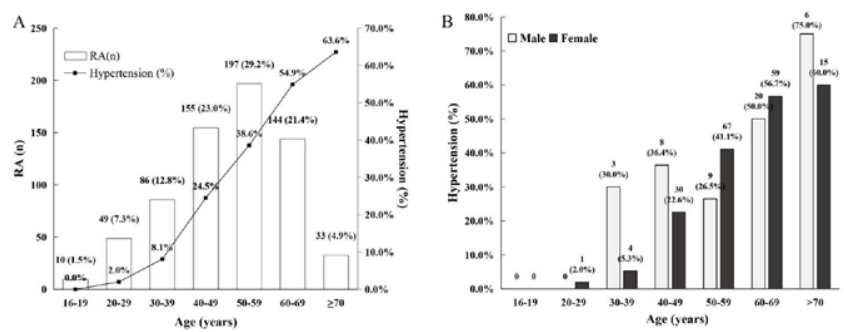

Figure 1. The prevalence of hypertension in different age and sex groups of RA patients.

Conclusion: Hypertension is the most common CV-related comorbidity in Chinese RA patients which is associated with hyperuricemia, dyslipidemia and fatty liver disease. Detection and management of hypertension and other CV-related comorbidities in RA patients should be emphasized.

\section{Fund program:}

National Natural Science Foundation of China (81801605, 81801606, 81971527); Guangdong Natural Science Foundation (2018A030313541 2018A030313690, 2019A1515011928); Guangzhou Science and Technology Program (201904010088); Guangdong Basic and Applied Basic Research Foundation (2020A1515110061); Guangdong Medical Scientific Research Foundation (A2018062)

Disclosure of Interests: None declared

DOI: 10.1136/annrheumdis-2021-eular.2078

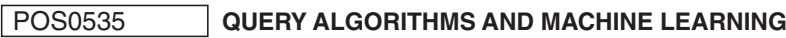 METHODS AS TOOLS TO IDENTIFY COMORBIDITIES IN LARGE-SCALE FREE-TEXT BASED FIELDS: A CASE-REPORT}

D. Rohrich ${ }^{1}$, T. Maarseveen ${ }^{2}$, A. de Boer ${ }^{1}$, C. van den Ende ${ }^{1}$, A. den Broeder ${ }^{1}$, C. Popa ${ }^{1}$, R. Knevel ${ }^{2}{ }^{1}$ Sint Maartenskliniek, Rheumatology, Ubbergen, Netherlands; ${ }^{2}$ Leiden University Medical Center (LUMC), Rheumatology, Leiden, Netherlands

Background: Inflammatory rheumatic conditions (IRC) are associated with comorbidity, two most important being cardiovascular diseases (CVD) and infections [1, 2]. A crucial initial step when proceeding with studying CVD and infections in these patients is identification of events. The large scale EHR datasets enable studies to assess low incident and clinically important events, but requires both accurate as well as efficient data extraction. Studying Electronic Health Records (EHR) using query-based algorithms (QBA) and machine learning algorithms (MLA) offers a valuable tool to screen large-scale collections for rare events, thus replacing resource intensive manual chart review.

Objectives: To explore the (comparative) usefulness of QBA and MLA to identify CVD and infection events in EHR free-text data in patients with chronic IRC.

Methods: To independently develop and validate the algorithms we used two EHR databases, i.e. a training set with psoriatic arthritis patients $(\mathrm{N}=977$, dataset $A$, Golden Standard) and a validation set of rheumatoid arthritis patients $(\mathrm{N}=1098$, dataset $\mathrm{B})$. Using both QBA and MLA, we aimed to identify (yes/no and timing) CVD and infections. We assessed the performances of the algorithms by calculating the specificity, sensitivity, positive predictive value (PPV) and the negative predictive value (NPV), respectively.

Results: In the final performance analysis on dataset $B$, both QBA and MLA showed a high performance in identifying CVD (sensitivity, specificity, PPV, NPV for QBA $((95 \% \mathrm{Cl})=0.69(0.66-0.72), 0.99(0.96-1.02), 0.84(0.81-0.87)$ $0.98(0.95-1.01))$ and for MLA (sensitivity $=0.69(0.66-0.72), 0.98$ (0.95-1.01), 0.68 (0.65-0.71), 0.98 (0.95-1.01), respectively) Infections showed similar performance (QBA sensitivity, specificity, PPV, NPV is $0.64(0.61-0.67), 0.96(0.93$ $0.99), 0.66$ (0.63-0.69), 0.96 (0.93-.0.99) and for MLA = 0.61 (0.58-0.64), 0.93 (0.90-0.96), 0.49 (0.46-0.52), 0.96 (0.93-0.99), respectively). For infections the specificity was slightly higher for QBA relative to MLA.

Conclusion: We found a consistent high performance of both the QBA and MLA algorithms for the identification of CVD and infections in our free text EHR of patients with chronic IRC (Table 1). The performance of QBA highly depends on the domain knowledge of the builders, which might allow it to outperform a Gold Standard. MLA is efficient as it does not require any domain knowledge, but its performance is restricted by the quality of the Gold Standard.

Table 1. Overlap and differences in event identification of the query based and machine learning based algorithms compared to the Gold Standard dataset B (chart review by specialized health care professional). The results of non-event and event identification by the algorithms is represented in numbers of events and percentages of the total non-event or event.

Dataset B Cardiovascular events

\begin{tabular}{|c|c|c|c|c|}
\hline & QBA - & ML - & QBA + & $M L+$ \\
\hline GS $-(\mathrm{N}=2123)$ & $2102(99.0 \%)$ & $2071(97.6 \%)$ & $21(0.99 \%)$ & $52(2.45 \%)$ \\
\hline $\mathrm{GS}+(\mathrm{N}=159)$ & $49(30.1 \%)$ & $49(30.1 \%)$ & $110(69.2 \%)$ & $110(69.2 \%)$ \\
\hline \multicolumn{5}{|c|}{ Total events GS =2282 } \\
\hline & QBA - & ML - & QBA + & ML + \\
\hline GS $-(N=2053)$ & 1979 (96.4\%) & 1910 (93.0\%) & 74 (3.6\%) & $143(7.0 \%)$ \\
\hline$G S+(N=228)$ & $83(36.4 \%)$ & $89(39.0 \%)$ & $145(63.6 \%)$ & $139(61.0 \%)$ \\
\hline Total events GS & & & & \\
\hline
\end{tabular}

(QBA = Query Based Algorithm, MLA = Machine Learning Algorithm, GS - = Non-event in Gold Standard dataset B, GS + = Event in Gold Standard dataset $B$ ) 